\title{
SU-8 PROCESS FOR BIOMEDICAL INSPECTION $\mu$-CARRIERS
}

\author{
P.-H. Chen ${ }^{+}$, P.-P. Ding ${ }^{*}$, and J.-S. Kuo ${ }^{*}$ \\ Department of Mechanical Engineering, National Taiwan University.
}

\begin{abstract}
The present study provides a novel carrier fabrication method for biomedical application. The SU-8 thick photoresist fabrication process is used to produce the $\mu$-carrier of $200 \mu \mathrm{m} \times 200 \mu \mathrm{m}$ with various thicknesses. These $\mu$-carriers will be used for the purpose of biomedical inspection after proper surface modification by chemicals. To quantify and qualify the immersed $\mu$-carriers in the inspected solution, specific marker is designed and fabricated on every $\mu$ carrier as identification according to the application. Hence, a precise control on the marker feature dimension will be crucial for the application. In addition, the thick resist layer uniformity is very important in defining the marker pattern on the surface of carrier. Besides, the sidewall profile is also crucial especially for the fabrication of $10 \mu \mathrm{m}$ through-hole markers on the carriers of $50 \mu \mathrm{m}$ to 100 $\mu \mathrm{m}$ thick. The orthogonal array experimental design method is employed to optimize the process parameters, including the soft bake, exposure, postexposure bake, and development parameters. Moreover, the effect of development with ultrasonic agitation is also investigated.
\end{abstract}

\section{INTRODUCTION}

Microelectromechanical system (MEMS) is an important enabling technology that has a deep impact across a number of industries, from mature transportation industry to emerging telecommunications and biomedical fields. One of the most regarded applications is to make daily used objects lighter and stronger to satisfy the demand for increased quality of life and superior health care. To increase the affordability, the two challenges facing by the emerging industry are to reduce the production cost by improving the fabrication yield, and to increase the diversity of product by the exploitation of new application areas. In particular, the development and improvement on the fabrication process will be one of the technology drivers to break the industry bottlenecks.

Biotechnology represents another evolving technologies recently. The advancements of micro and nano technologies in the past decades dramatically accelerate the development and broaden the application of biotechnology. Potential applications include the biochemical test, pharmaceutical industry, medical diagnosis, agrochemical products, environmental monitoring system and other industries. As a result, the integration between biotechnology and micro or nano fabrication has become an inevitable trend. The development of biochips for genetic, toxicological, protein, forensic, and biochemical applications, is a noticeable result. Typically, a biochip is a host of micro arrays fabricated on a substrate. To achieve higher throughput and rapid detection, the arrangement might allow many tests to be performed simultaneously. While a computer chip performs millions of operations in one second, a biochip performs thousands of biochemical reactions in a few seconds.

\footnotetext{
${ }^{+}$Professor

* Ph D student
} 


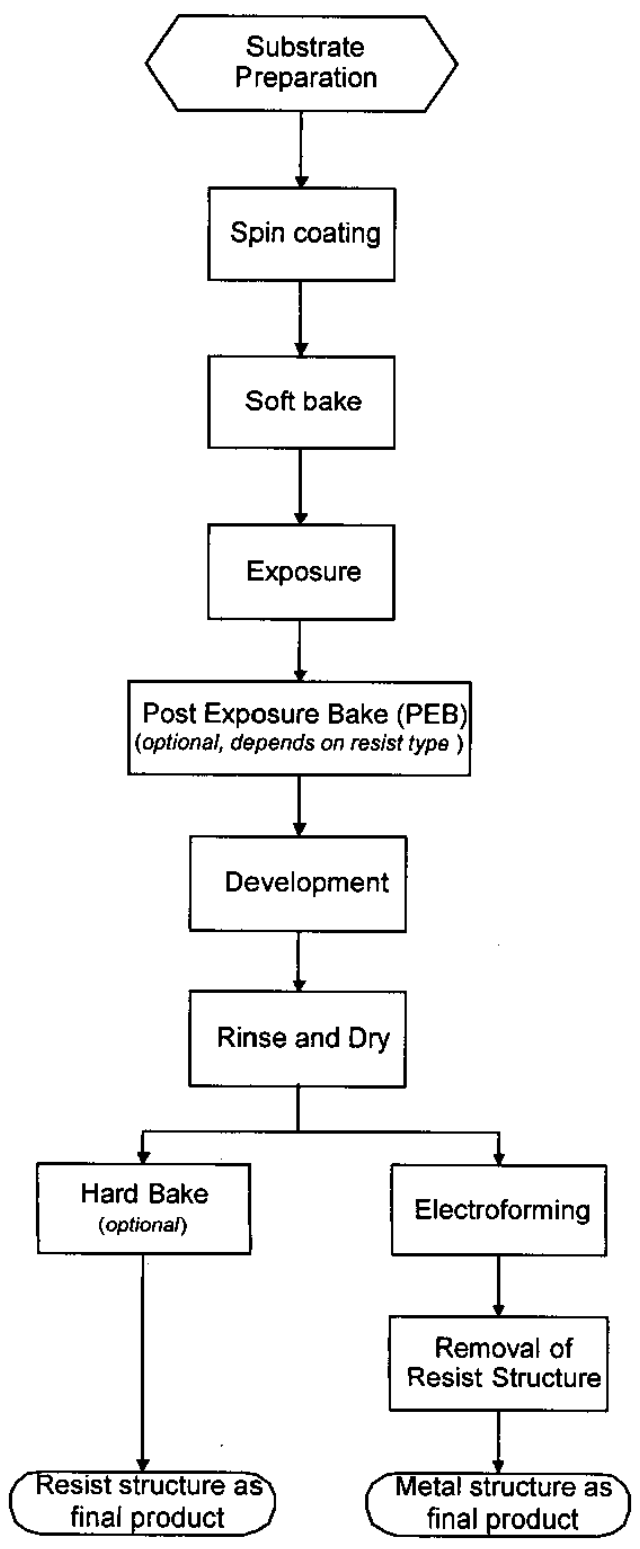

Figure 1 Typical UV-LIGA process.

The present study provides a novel carrier fabrication method for biomedical application. The SU-8 thick photoresist fabrication process is used to produce the $\mu$-carriers. These $\mu$-carriers will be used for the purpose of biomedical inspection after proper surface modification by chemicals or reagents. To

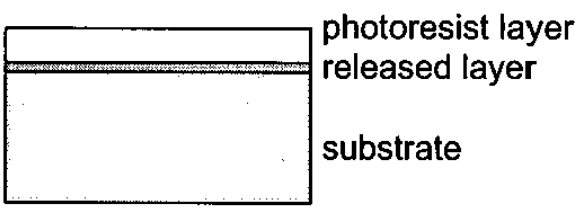

(a) photoresist coating

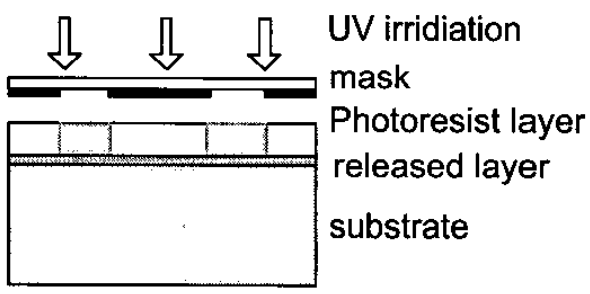

(b) photolithography

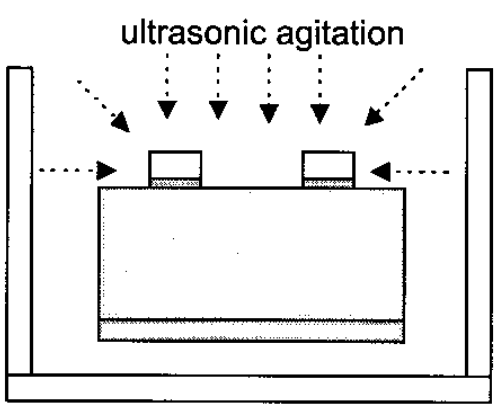

(c) development

Figure 2 Photolithography process for SU-8 resist: (a) spin coating of SU-8 released layer and SU-8 resist, soft bake, (b) exposure, postexposure bake, (c) ultrasonic-assisted development.

quantify and qualify the immersed $\mu$-carriers in the inspected solution, specific marker is designed and fabricated on every $\mu$-carrier as identification according to the purpose of application. This method prevents the possibility of contamination issue due to the design and compact arrangement of microarrays on most commercially available biochips. Meanwhile, the cost can be lowered since the user can decide the number or type of $\mu$-carriers according to the amount of inspected solution or the purpose of detection.

\section{FABRICATION PROCESS}


Chen et al.

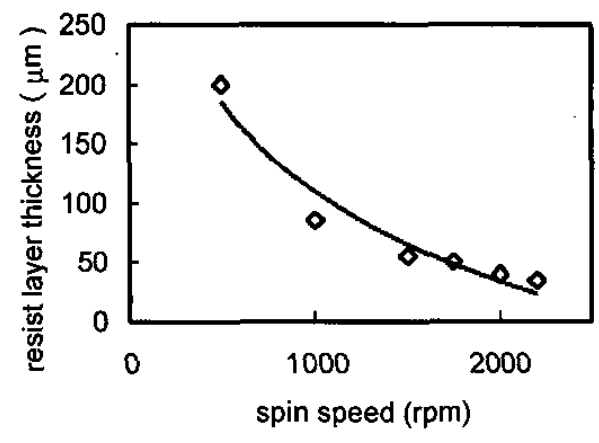

Figure 3 Thickness of coated resist layer under various spin speed.

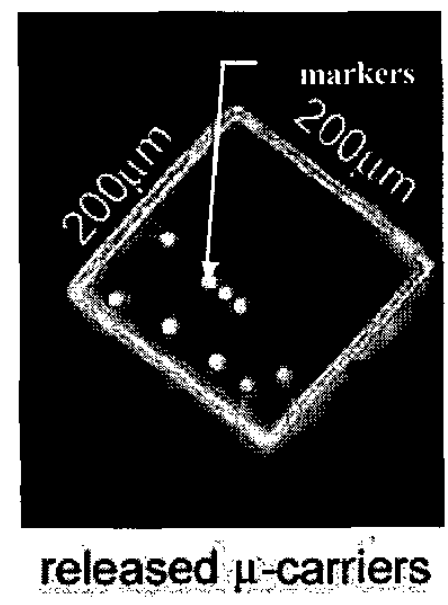

Figure 4 Released $\mu$-carriers by ultrasonicassisted development.

Due to the fact that the construction of synchrotron is extremely expensive, the advancement of the LIGA technology is restricted. In order to create powerful but low cost micromachining technique, cost affordable alternative irradiation sources have been developed and used instead of synchrotron radiation. The process performed by using alternative techniques, such as UV photolithography, reactive ion etching (RIE), excimer laser ablasion and others, is generally called the LIGA-like process. Besides, there are a number of commercially available photoresists that allow thick resist layer fabrications, such as
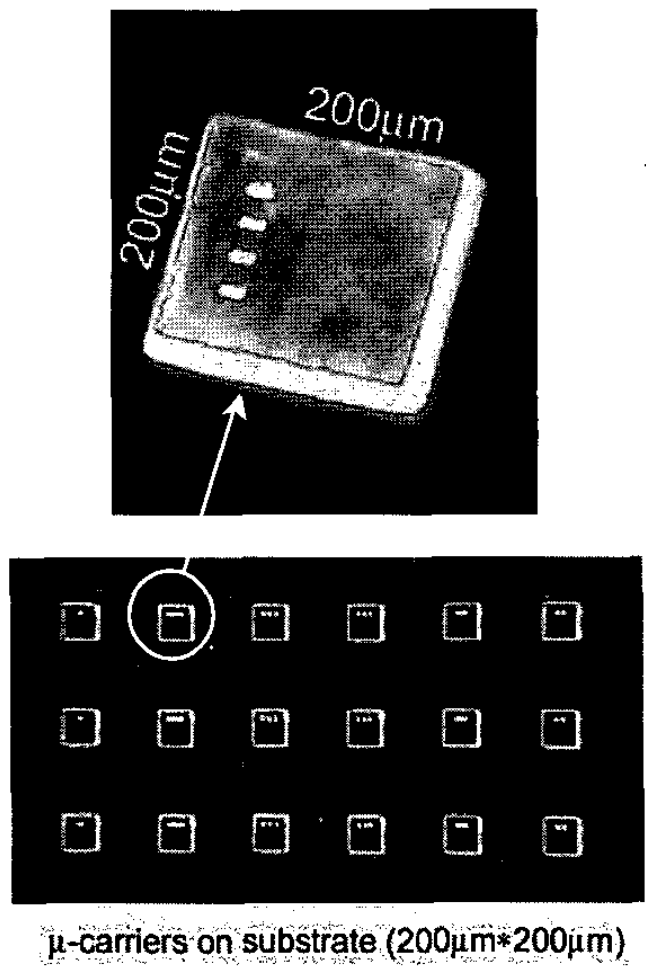

Figure 5 Microfabricated SU-8 $\mu$-carriers with markers.

positive tone ma-P series (Micro Resist Technology). The development on the relatively new photoresist, such as SU-8, for high aspect ratio photolithography is also an alternative called the UV-LIGA process. The typical UV-LIGA process is shown in Figure 1. SU- 8 series resist is an epoxy based announced by IBM that is potential to fabricate thick microstructures in single-step coating. Among the commercially available resists in the market, the SU- 8 series resist has the highest epoxide functionality [1]. Upon exposure, the resist layer coated on a substrate will form a highly structured crosslinked matrix. The low molecular weight of the resin provides high contrast, excellent solubility, and planarizing capability, while the high epoxy functionality provides sensitivity.

A uniform thick photoresist layer can be best obtained in a closed coating environment by using a coater with a co-rotating cover. A Karl Suss RC-8 spin coater was used for spin coating of thick resist 
layer in the present work. The coating process will be performed in a closed, solvent saturated environment, which prevents the evaporation of resist, between the substrate and the co-rotating cover. In the present work, the edge bead of resist hyer (MicroChem, SU8-50) was removed to control the film thickness. To obtain released $\mu$-carriers, the process procedures for the SU-8 series resist includes substrate preparation, resist coating, soft bake, exposure, post exposure bake, development, rinse, released of $\mu$-carriers and dry [2], as shown in Figure 2.

\section{RESULTS AND DISCUSSIONS}

In order to control the surface area of each $\mu$-carrier, a uniform resist layer is critical. An access amount of resist was dispensed on a 4 inch cleaned substrate to ensure a uniform target film thickness over the entire surface (Figure 3). For the present work, specific marker was designed and fabricated on every $\mu$ carrier as identification according to the purpose of application. Hence, the dimension of the marker will be crucial for the application. Resist layers of $50 \mu \mathrm{h}$ and $100 \mu \mathrm{m}$ were chosen. For the design of markers arrangement, line width between $8 \mu \mathrm{m}$ and $14 \mu \mathrm{m}$, and through-holes of circular, rectangular and square shape were fabricated.

As mentioned previously, a spin coater with a co-rotating cover should be used to ensure the uniforminty of thick resist layers. Hence, the thickness variation can be controlled within $5 \%$ for a batch of $\mu$-carriers with various marker arrangements. For all samples in the present work, the edge bead was removed after spin coating to ensure a good contact between mask and resist can be obtained in contact lithography. Besides, the edge bead effect of SU-8 resist can be slightly eliminated by soft bake procedure due to ability to self-planarize during baking.

In practical application, photoresist has been shown to be sensitive to the variations of process parameters. The process parameters vary in a wide range and no standard process parameter has been demonstrated as the most significant factors in achieving thick microstructures with vertical sidewall. To optimize the process parameters, the Taguchi's method was employed in the present work $[3,4]$

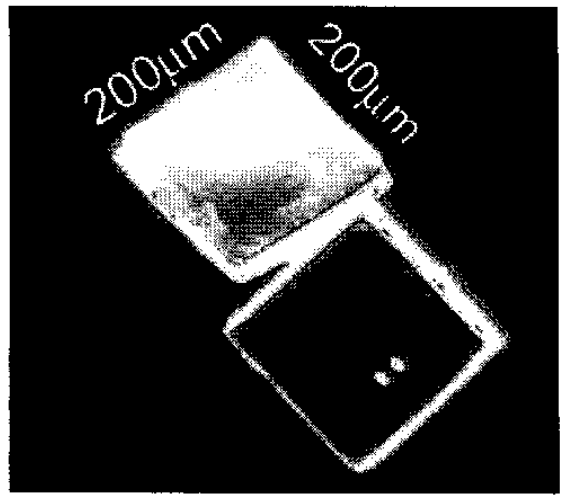

Figure 6 Released $\mu$-carriers with various thicknesses.

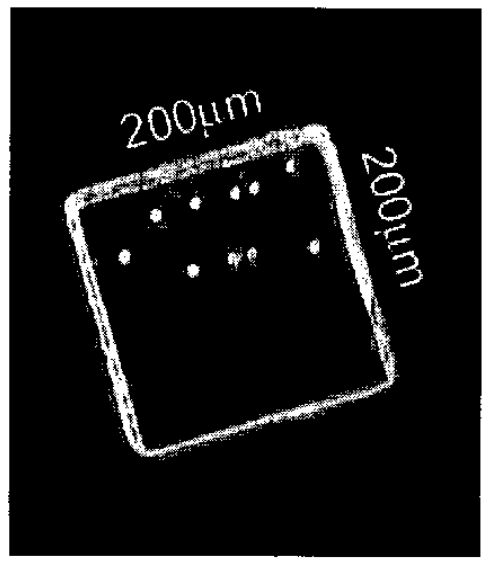

Figure 7 The optimum dimension of throughhole marker for $\mu$-carriers of $50 \mu \mathrm{m}$-thick is circular hole with diameter of $10 \mu \mathrm{m}$.

Figure 4 shows a released $\mu$-carrier fabricated by SU-8-50. The thickness of $\mu$-carriers on substrate shown in Figure 5 is $50 \mu \mathrm{m}$. As shown in Figure 6, the released $200 \mu \mathrm{m} \times 200 \mu \mathrm{m} \mu$-carriers remained undestroyed after ultrasonic agitation [5]. Finally, the optimum marker was found to be circular throughhole with minimum diameter of $10 \mu \mathrm{m}$.

\section{CONCLUSIONS}




\section{Chen et al.}

A $\mu$-carrier fabricated by SU-8-50 was performed for biomedical application. The SU-8 thick photoresist fabrication process is used to produce the $\mu$-carrier of $200 \mu \mathrm{m} \times 200 \mu \mathrm{m}$ with various thicknesses. To ensure thickness uniformity of $\mu$-carriers, the coating process was performed in a closed, solvent saturated environment, which prevents the evaporation of resist between the substrate and the co-rotating cover. The thickness variation from substrate to substrate can be controlled within $5 \%$ by using the Karl Suss RC-8 spin coater.

Specific marker was designed and fabricated on every $\mu$-carrier as identification according to the application. The conclusions can be summarized as follows:

(a) For both $50 \mu \mathrm{m}$ and $100 \mu \mathrm{m} \mu$-carriers fabricated in the present study, the optimum marker was found to be circular through-hole with minimum diameter of $10 \mu \mathrm{m}$.

(b) To release the $\mu$-carriers from the substrate and prevent the over-adhesion problem, an additional SU-8 released layer was coated on the substrate in the present study. As a post-development procedure, an ultrasonic-assisted agitation was employed to release the $\mu$-carriers.

\section{ACKNOLEDGMENT}

The authors deeply appreciate the financial support by NSC under the grant number 90-2218-E-002-032, equipment support by NSC MEMS Research Center, and technical support by GeneMaster Lifesciences, Taiwan. The work in this study could not be achieved without their support.

\section{REFERENCES}

[1] H. Lorenz, M. Despont, N. Fahrni, N. LaBianca, P. Renaud, and P. Vettiger, "SU-8: A Low Cost Negative Resist for MEMS," J. Micromech. Microeng., Vol. 7, pp. 121-124, 1997.

[2] J. M. Shaw, J. D. Gelorme, N. C. LaBianca, Conley W. E., and S. J. Holmes, "Negative Photoresists for Optical Lithography," IBM $J$. Res. Develop., Vol. 41, No. 1/2, pp. 81-94, 1997.

[3] J. Zanghellini, A. El-Kholi, and J. Mohr, "Development Behavior of Irradiated Microstructures," Microelectronic Engineering, Vol. 35, pp. 409-412, 1997.
[4] G. Bleidiessel, G. Gruetzner, F. Reuther, S. Fehlberg, B. Loechel, and A. Maciossek, "Dependence of The Quality of Thick Resist Structures on Resist Baking," Microelectronic Engineering, Vol. 41/42, pp. 433-436, 1998.

[5] J. Zanghellini, S. Achenbach, A. El-Kholi, J. Mohr, and F. J. Pantenburg, "New Development Strategies for High Aspect Ratio Microstructures," Microsystem Technologies, Vol. 4, pp. 94-97, 1998. 